\title{
The impact of genetic modifiers on variation in germline mutation rates within and among human populations
}

\author{
William R. Milligan a, 1, Guy Amster a, b and Guy Sella a, c, 1
}

a Department of Biological Sciences, Columbia University, New York, NY 10027

b Flatiron Health Inc., 233 Spring St New York, NY 10013

c Program for Mathematical Genomics, Columbia University, New York, NY 10032

1 To whom correspondence should be addressed: wm2377@columbia.edu or gs2742@columbia.edu

\section{Abstract}

2 Mutation rates and spectra differ among human populations. Here, we examine whether this

3 variation could be explained by evolution at mutation modifiers. To this end, we consider

4 genetic modifier sites at which mutations, "mutator alleles", increase genome-wide mutation

5 rates and model their evolution under purifying selection due to the additional deleterious mutations that they cause, genetic drift, and demographic processes. We solve the model analytically for a constant population size and characterize how evolution at modifier sites impacts variation in mutation rates within and among populations. We then use simulations to study the effects of modifier sites under a plausible demographic model for Africans and

10 Europeans. When comparing populations that evolve independently, weakly selected

11 modifier sites $\left(2 N_{e} s \approx 1\right)$, which evolve slowly, contribute the most to variation in mutation

12 rates. In contrast, when populations recently split from a common ancestral population, 13 strongly selected modifier sites $\left(2 N_{e} s \gg 1\right)$, which evolve rapidly, contribute the most to

14 variation between them. Moreover, a modest number of modifier sites (e.g., 10 per mutation 15 type in the standard classification into 96 types) subject to moderate to strong selection $16\left(2 N_{e} S>1\right)$ could account for the variation in mutation rates observed among human 17 populations. If such modifier sites indeed underlie differences among populations, they 18 should also cause variation in mutation rates within populations and their effects should be 19 detectable in pedigree studies. 


\section{Introduction}

21 Germline mutations are the ultimate source of genetic variation, fueling the evolutionary

22 process. Yet mutation rates themselves also vary and evolve (Elango et al. 2006; Elango et

23 al. 2009; Lynch 2010; Sayres et al. 2011; Scally and Durbin 2012; Ségurel et al. 2014; Harris

24 2015; Harris and Pritchard 2017; Mathieson and Reich 2017). For example, mutation rates

25 among vertebrates differ by almost an order of magnitude (Kong et al. 2012; Venn et al.

26 2014; Uchimura et al. 2015; Feng et al. 2017; Lindsay et al. 2019; Harland et al. 2017), and

27 the mutation spectrum differs among primate species (Moorjani et al. 2016; Harris and

28 Pritchard 2017; Goldberg and Harris 2019).

29 Mutations at loci that modify germline mutation rates, such as in genes involved in DNA

30 replication and repair, can change the rate and spectrum of mutations (Drake 1993; Aarnio

31 et al. 1999; Sasani et al. 2021). Such "mutator alleles" are subject to purifying selection

32 when they increase the burden of deleterious mutations in individuals who carry them

33 (Kimura 1967; Kondrashov 1995; Lynch 2008). Their population frequencies are also

34 affected by genetic drift and demographic processes, such as changes in population size.

35 Therefore, modifiers of germline mutations evolve under an interplay between mutation,

36 purifying selection, genetic drift, and demographic processes, which generates variation in

37 rates and spectrum of germline mutations among individuals, populations, and species.

Differences in the efficacy of selection against mutator alleles could explain variation in mutation rates among evolutionary distant taxa. The strength of selection acting on a mutator allele (quantified by its selection coefficient $s$ ) should be proportional to its effect

41 on mutation rates (Kimura 1967; Kondrashov 1995; Dawson 1998). Species with effective

42 population sizes, $N_{e}$, cannot effectively select against mutators with $2 N_{e} S \ll 1$ (Crow and

43 Kimura 1970) and thus species with smaller $N_{e}$ are expected to have higher mutation rates

44 (Kimura 1967; Lynch 2008; Lynch 2010; Sung et al. 2012). Variation in $N_{e}$ may then explain

45 much of the variation in mutation rates among taxa (Lynch 2010; Leffler et al. 2012; Sung

46 et al. 2012) as well as aspects of genome architecture such as variation in genome size

47 (Lynch and Conery 2003; Lynch 2007). 
Mutation rates have also been observed to vary substantially over shorter evolutionary timescales, in particular among human populations (Harris 2015; Moorjani et al. 2016; Harris and Pritchard 2017; Mathieson and Reich 2017; Narasimhan et al. 2017; Aikens et al. 2019; Speidel et al. 2019; Goldberg and Harris 2019). When mutations are categorized into 96 different types based on ancestral allele, derived allele, and 5' and 3' flanking nucleotides (e.g., GAC $\rightarrow$ GTC), roughly one-third of these types show significant differences in mutation rates between pairs of continental populations (Harris and Pritchard 2017). Notably, rates of TCC $\rightarrow$ TTC mutations were estimated to have temporarily increased by 50-100\% between 5,000-30,000 years ago in Europeans, whereas these rates have remained approximately constant in Africans (Harris 2015; Harris and Pritchard 2017; Mathieson and Reich 2017; Speidel et al. 2019). Here, we consider how such a large and pulse-like increase in a mutation rate could arise.

Multiple explanations have been proposed for these observations, which are not mutuallyexclusive: changes to life history traits that affect mutation rates and spectra, changes in environment that led to differences in mutagen exposures, and evolution at mutation rate modifiers (Harris 2015; Harris and Pritchard 2017; Mathieson and Reich 2017; Carlson et al. 2020; Chintalapati and Moorjani 2020; Macià et al. 2021). Differences in life history traits (notably in generation times) among populations could have generated differences in multiple mutation rates, including the rate of TCC $\rightarrow$ TTC mutations (Macià et al. 2021). We may be able to learn whether changes in generation times plausibly explain the differences in mutations among populations by testing if these differences align with the effects of parental ages on de novo mutation rates in human pedigree studies (Z. Gao and M. Przeworski, personal communication). Similarly, some environmental mutagens preferentially increase $\mathrm{C} \rightarrow \mathrm{T}$ mutation rates, such as alkylating agents that increase $\mathrm{G} \rightarrow \mathrm{A}$ mutation rates (Mathieson and Reich 2017) or UV-damage, which has been associated with increased TCN $\rightarrow$ TTN and CCN $\rightarrow$ CTN mutation rates in irradiated cells (Drobetsky and Sage

74 1993; Marionnet et al. 1995) and melanomas (Alexandrov et al. 2020). Additionally,

75 humans populations faced widespread environmental changes during the relevant time

76 period, which may have changed their exposures to such mutagens (Firestone et al. 2007;

77 Broughton and Weitzel 2018; Pino et al. 2019). However, we know little about how 
relevant exposures changed over time or how various mutagens affect germline mutation rates. Consequently, it is difficult to evaluate whether changes in environment plausibly explain variation in germline mutation rates.

81 Alternatively, variation in mutation rates among human lineages could reflect evolution at

82 loci that modify germline mutation rates (Harris and Pritchard 2017). Modifier loci that

83 cause variation among populations should also cause variation within populations, which

84 suggests that we may be able to identify their effects in datasets of de novo mutations in

85 pedigrees, genetic variation data from unrelated individuals (see, e.g., Seoighe and Scally

86 2017), or by quantitative trait locus (QTL) mapping (see, e.g., Sasani et al. 2021). However,

87 to understand whether this explanation is plausible-that is whether evolution at modifier

88 loci could explain observed population differences-requires a model of mutator allele

89 dynamics.

90 Our goal is therefore to describe how the interplay of mutation, selection, drift, and

91 demographic processes at loci that modify germline mutation rates shapes variation in

92 rates among and within populations. Specifically, we ask: 1) how the number and effect

93 sizes of modifier loci affect their contribution to variation among and within populations;

94 2) whether evolution at modifier loci could generate the kind of variation observed among

95 human populations, and 3) how we might identify the effects of mutator alleles in datasets

96 of de novo mutations in pedigrees.

\section{The Model}

98 We assume that an individual's genotype consists of two parts: $L$ strongly selected, biallelic 99 sites that determine fitness and $M$ biallelic modifier sites that determine the mutation rate.

100 Specifically, we assume that fitness combines multiplicatively over the selected sites and

101 that each deleterious allele reduces fitness by a factor of $(1-h s)$, where $h s>0$. Thus, an

102 individual carrying $i$ deleterious alleles has absolute fitness $(1-h s)^{i}$. Our main results

103 should hold under more general fitness models (e.g., with a distribution of selection effects)

104 and depend primarily on the compound parameter $2 L \cdot E(h s)$. In our mutation model, we 
105 assume a baseline mutation rate of $u_{0}$ per site per generation, which is the mutation rate

106 obtained with optimized replication and repair machinery (i.e., one that minimizes

107 mutations). We further assume that at each of the $M$ modifier sites, one allele is optimal

108 (i.e., it does not increase mutation rates), while the other, mutator allele increases the

109 mutation rate by $\phi$. Thus, an individual carrying $m$ mutator alleles will have mutation rate

$$
u=u_{0}+m \phi \text {, }
$$

110 where we assume the effects of mutator alleles are additive across and within sites. Again,

111 our main results should hold under more complex mutation models, e.g., with a

112 distribution of mutator allele effect sizes.

113 We first model the evolution of mutation rates in a diploid, panmictic population of constant

114 size $N$, with non-overlapping generations. In each generation, individuals are randomly

115 chosen as parents with probabilities proportional to their fitness (i.e., according to Wright-

116 Fisher sampling with fertility selection). These individuals then produce gametes with free

117 recombination among selected and modifier sites, Mendelian segregation, and mutations at

118 both the modifier and selected sites. We assume that the mutational input per generation

119 per selected site is sufficiently low $(2 N u \ll 1)$ and that deleterious alleles are sufficiently

120 rare $(2 N h s \gg 1)$ such that we can apply the infinite sites approximation and ignore reverse

121 mutations. The number of deleterious alleles added per gamete per generation is then

122 Poisson distributed with mean $\mathrm{Lu}$. We further assume that the mutation rate at modifier sites

123 per generation, $\mu$, is symmetric among alleles and constant (i.e., not affected by mutator

124 alleles). We later show that relaxing this assumption does not affect our qualitative results

125 but may lead to transient surges in mean mutation rates (Fig. S5); we expect this effect will

126 be negligible when individual modifier sites only affect the mutation rate corresponding to a

127 subset of mutation types, as the opportunity for non-linear interactions among modifier sites

128 will be greatly reduced. Finally, we assume that gametes combine randomly to produce the

129 next generation.

130 We later consider a version of this model under a scenario that mimics a widely-used

131 demographic history inferred for European and African populations by Schiffels and 
132 Durbin (2014); hereafter the S\&D demographic history (see Fig. S4 for details). Instead of a

133 single, constant-size population, we consider an ancestral population that splits into two

134 independently-evolving populations, where population sizes change over time. For this

135 scenario, we focus on variation among populations in mutation rates affecting one of the 96

136 potential mutation types, such as TCC $\rightarrow$ TTC (which have decreased $L$ and $M$ compared to

137 mutators that affect all mutation types jointly).

138 Simulations. We compare our analytic approximations for the mean and variance of

139 mutation rates in a constant-size population with results from simulations that implement

140 this model across a wide range of effect sizes $\phi$ and number of modifier sites $M$.

141 Simulations with large $M$ become computationally prohibitive, so we restrict ourselves to

142 simulations with $M \leq 10,000$. In each simulation, we initialize mutator allele frequencies

143 by sampling from their stationary distribution (see Results) and the number of deleterious

144 alleles carried by individuals by sampling from a Poisson distribution with mean $2 L \hat{u} / h s$

145 (Felsenstein 1974), where $\hat{u}$ is the expected mutation rate (see Results). Then, we apply a

146 burn-in period of $10 \mathrm{~N}$ generations such that the population has attained mutation-

147 selection-drift balance at both selected and modifier sites. Each simulation runs for $200 \mathrm{~N}$

148 generations. The number of replicates for each combination of parameters is the maximum

149 of 10 and the number needed such that the relative errors in the estimated expectation and

150 variance in mutator allele frequency and expected heterozygosity at modifier sites is less

151 than 25\%. We estimate the expectation and variance in mutator allele frequency and the

152 expected heterozygosity at modifier sites based on frequencies sampled every $N$

153 generations and by averaging across all replicates; we calculate heterozygosity assuming

154 Hardy-Weinberg equilibrium (i.e., $\pi=2 q(1-q)$ ). Standard error in these quantities is

155 calculated as $\sqrt{\sigma^{2} / n}$, where $\sigma^{2}$ is the variance in the estimated quantity across replicates

156 and $n$ is the number of replicates. Increasing the intervals used has little effect on our

157 results. We convert from moments of mutator allele frequencies to moments of mutation

158 rates using simple transformations (Eqs. 13-15). 
159 We also use simulations to investigate whether evolution at modifier sites could account

160 for observed differences in mutation rates between European and African populations.

161 Specifically, we assume the same model as described above with the S\&D demographic

162 history of European and African populations (Fig. S4). Here, we separate mutations into 96

163 different yet equivalent types - corresponding to the 96 types analyzed in previous work

164 (Harris 2015; Harris and Pritchard 2017; Mathieson and Reich 2017; Speidel et al. 2019) -

165 with the rate of each type being affected by its associated $M$ modifier sites with effect size

$166 \phi$. For each mutation type, we simulate mutation rate trajectories - the mean mutation rate

167 in a population recorded each generation - which we later use to assess variation in mean

168 mutation rates among populations.

169 Generating large samples of mutation rate trajectories for different combinations of $\phi$ and

$170 \mathrm{M}$ is computationally prohibitive. Therefore, given $\phi$ and $M$, we generate mutation rate

171 trajectories by sampling $M$ allele trajectories from an ensemble of 15,000 allele trajectories

172 at modifier sites with effect size $\phi$. This ensemble is simulated as follows: 1) We run 15

173 replicates of a simulation with $M=10^{3}$ modifier sites with effect size $\phi$, where other

174 parameters take plausible values for humans (see Table 1). 2) The initial mutator allele

175 frequency at each site is sampled from our analytic approximation of the stationary

176 distribution with an effective population size of $N=1.4 \cdot 10^{4}$, the estimate for $7 \cdot 10^{4}$

177 generations ago under the S\&D demographic history. 3) We run the simulation for a burn-

178 in period of $10^{4}$ generations with a constant population size of $N=1.4 \cdot 10^{4}$. 4) We

179 simulate evolution at the modifier sites for $6 \cdot 10^{4}$ generations in the ancestral population

180 followed by $10^{4}$ generations in each of the two descendant populations separately, where

181 population sizes change according to the S\&D demographic history. To generate mutation

182 rate trajectories, we sample $M$ of the resulting allelic trajectories, sum their contributions

183 to mean mutation rates in each generation, and add a baseline mutation rate $u_{0}$. The $u_{0}$ is

184 chosen such that our approximation for the expected mutation rate in a population of

185 constant size $N=1.4 \cdot 10^{4}$ matches a given expected mutation rate $\hat{u}$ (see Table 1 ). Our

186 simulations exclude the most recent 60 generations, where population sizes are very large.

187 Neglecting this short period should have a negligible effect on our results as there is little

188 opportunity for drift or mutations at modifier sites. Additionally, our results are plausibly 
insensitive to the minor statistical dependencies among allelic trajectories in our simulations and to our sampling approach more generally (Fig. S10).

Tests for variation in mutation rates between populations. We use two measures to

192 investigate whether evolution at modifier sites could generate the differences in mutation

193 rates observed between European and African populations. The first mimics the

194 enrichment analysis of Harris and Pritchard (2017), who asked if the fraction of

195 segregating sites corresponding to a given mutation type differed among populations.

196 Specifically, they measured enrichments of a given mutation type in approximately 500

197 individuals of European ancestry and 500 of African ancestry using the statistic:

$$
r_{F}=\frac{F(\text { European })}{T(\text { European })} / \frac{F(\text { African })}{T(\text { African })},
$$

199 where $F(X)$ denotes the number of segregating sites corresponding to the focal type in 200 population $X$ and $T(X)$ denotes the total number of segregating sites in population $X$. This 201 statistic is heavily weighted towards recent differences in mutation rates (see Fig. S8).

202 We mimic their analysis for each combination of $\phi$ and $M$. First, we use msprime (Kelleher

203 et al. 2016) to simulate the ancestral recombination graph (ARG) assuming the S\&D

204 demographic history, a sample size of 500 individuals in each population, a genome size of $2053 \times 10^{9}$ basis divided into 100 equally sized chromosomes, and a uniform recombination 206 rate of $2 \cdot 10^{-8}$ per bp per generation. Second, for a given set of 96 mutation rate 207 trajectories (see Simulations), we drop mutations corresponding to each mutation type on 208 the ARG and obtain a polymorphism dataset that mimics the one used by Harris and 209 Pritchard (2017) (see SI Section 4). Third, we use this dataset to calculate the enrichment 210 of each mutation type, $r_{F}$, and record the largest enrichment among the 96 . Fourth, we 211 repeat the second and third steps for each of the $10^{5}$ sets of mutation rate trajectories to 212 obtain an empirical distribution of mutation rate enrichments for a given $\phi$ and $M$. We use 213 the same simulated ARG throughout, but variation in the ARG is negligible (Fig. S7) and its 214 effects on our analysis is largely removed by the ratio for estimating mutation rate 215 differences, $\mathrm{F} / \mathrm{T}$. 
216 For our second measure of variation in mutation rates, we consider the average mutation

217 rate across time intervals, which Speidel et al. (2019) estimated for human populations.

218 Specifically, we calculate the average mutation rate in 14 non-overlapping log-sized time

219 intervals along our simulated mutation rate trajectories (seven per population during the

$22010^{4}$ generations since they split, with the times demarking the intervals being $60,124,258$,

$221537,1116,2318,4814$, and 10000 generations). We then test for the peak-like behavior

222 observed in human populations, as follows: 1) We define an interval as elevated if its

223 average rate is at least $10 \%$ greater than the expected rate or peaked if the average is $50 \%$

224 greater than expected. We later relax this definition such that the average rate must be

$22550 \%$ greater than the expected rate for an interval to be elevated (Fig. S11), 2) We classify

226 mutation rate trajectories into three categories: peak-like if one interval is peaked and none

227 of the other intervals are elevated; multi-elevated if multiple intervals are elevated; or not-

228 peaked if none of the intervals are peaked and at most one is elevated. 3) We then classify $a$

229 set of mutation rate trajectories as: peak-like if it includes at least one peak-like trajectory

230 but none of the trajectories are multi-elevated; multi-elevated if one or more of the

231 trajectories are multi-elevated trajectories; or not-peaked if all trajectories are not-peaked.

232 Finally, we estimate the probabilities that a set of mutation rate trajectories would be peak-

233 like, multi-elevated and not-peaked for different values of $M$ and $\phi$.

234 Model parameters. The parameters of the model and the values we use for them are

235 detailed in Table 1. In our analyses, we vary the effect size, $\phi$, and number of modifier sites,

$236 M$, while setting other parameters to plausible values for humans. We also investigate the

237 sensitivity of our results to changes in these parameter values (Fig. S2).

238 First, consider mutation parameters. Estimates of the average point mutation rate in

239 humans are $\sim 1.25 \cdot 10^{-8}$ per site per generation (Kong et al. 2012). Accordingly, we

240 assume that the mutation rate at modifier sites is on average $\mu=1.25 \cdot 10^{-8}$ per

241 generation and that the expected mutation rate at selected sites is $E(u)=\hat{u}=1.25 \cdot 10^{-8}$

242 per generation. When we consider a specific mutation type (e.g., TCC $\rightarrow$ TTC), we use a

243 third of this $\hat{u}$ estimate. As we detail in Results, the expected mutation rate per site given $M$

244 modifier sites with effect size $\phi$ is $\hat{u}=u_{0}+M \cdot 2 E(q) \phi$, where $E(q)$ is the expected 
245 mutator allele frequency. We use the estimated value of $\hat{u}$ to calculate the maximal number

246 of modifier sites with a given effect size $\phi, M^{*}(\phi)$, and to determine the baseline mutation

247 rate $u_{0}$ given $M$ modifier sites with a given effect size $\phi$.

248 Second, consider selection parameters. Selected sites affect mutator allele dynamics

249 through the compound parameter Lhs. Estimates of the proportion of the human genome

250 subject to purifying selection range from 7-9\% (Ponting and Hardison 2011; Ward and

251 Kellis 2012; Kellis et al. 2014; Rands et al. 2014) corresponding to $\sim 2.1-2.7 \times 10^{8}$ selected

252 sites. We do not currently have good estimates for the average strength of selection at

253 these sites. Assuming that the average selection coefficient is within a factor of two of

$254 E(h s) \approx 5 \cdot 10^{-4}$, such that $2 N E(h s) \approx 20$ assuming $N_{e} \approx 2 \cdot 10^{4}$ (Schiffels and Durbin

255 2014). This suggest that genome-wide $2 L E(h s) \approx 2.4 \times 10^{5}$. We use this value when we

256 consider modifier sites affecting the total mutation rate and 1/32 of this value when we

257 consider the mutation rate at one of 32 potential trinucleotide sequence motifs (e.g., TCC).

258 In our analyses, we keep these selection parameters constant (See Table 1) and explore

259 how varying mutator allele effect size $\phi$, which varies the strength of selection against

260 mutator alleles, impacts their dynamics. Alternative values of parameters yield similar

261 results (Fig. S2).

262 Lastly, consider the population size. For the model with a constant population size, we use

$263 N_{e} \approx 2 \cdot 10^{4}$ (Schiffels and Durbin 2014). For the results corresponding to variation

264 between African and European populations, we use the S\&D demographic history (Fig. S4).

265 When we test our analytic predictions for the model with a constant population size against

266 simulations, we use a smaller population size of $N_{e}=10^{3}$ for computational efficiency. In

267 this case, we scale other parameters such that their population scaled values, i.e., $2 N_{e} \overline{h s}$,

$2682 N_{e} \mu, 2 N_{e} \hat{u}$, and $2 L h s$, retain plausible values (i.e., those corresponding to $N_{e} \approx 2 \cdot 10^{4}$ ). 


\begin{tabular}{|c|l|c|}
\hline $\begin{array}{c}\text { Parameter } \\
\phi\end{array}$ & \multicolumn{1}{|c|}{ Meaning } & \multicolumn{1}{c|}{$\begin{array}{c}\text { Value } \\
\text { Increase in mutation rate per mutator allele }\end{array}$} \\
\hline$M$ & Number of modifier sites & Varied but bounded given $\hat{u}$ \\
\hline$\hat{u}$ & Expected mutation rate at selected sites & $1.25 \cdot 10^{-8}$ or $1.25 / 3 \cdot 10^{-8}$ \\
\hline$u_{0}$ & Baseline mutation rate & Fixed given $M, \phi, \hat{u}(\mathrm{Eq} .13)$ \\
\hline$\mu$ & Mutation rate at modifier sites & $1.25 \cdot 10^{-8}$ \\
\hline$L h s$ & Cumulative fitness effect of mutations at & $1.2 \cdot 10^{5}$ or $1.2 \cdot 10^{5} / 32$ \\
\hline$N_{e}$ & selected sites affected by mutator allele & $2 \cdot 10^{4}$ or taken from \\
& Effective population size & Schiffels and Durbin $(2014)$ \\
\hline
\end{tabular}

Table 1. Model parameters and their values used.

270 The software used for all simulations, analytic approximations, and figures can be found at 271 https://github.com/sellalab/Mutator.

\section{Results}

273 Mutation rates at steady state. We begin by considering the expectation and variance of 274 mutation rates in a population of constant size at steady state. First, we approximate the 275 expected selection coefficient against a mutator allele with a given effect size $\phi$. Second, we 276 rely on that selection coefficient to derive a diffusion approximation for the stationary 277 distribution of mutator allele frequency. Third, we rely on the stationary distribution to 278 calculate the expectation and variance of the mutation rate in models with many modifier 279 sites and validate our results against simulations. These results provide an understanding 280 of how mutation-selection-drift balance at modifier sites affects mutation rates, which later 281 informs our analysis of the more complicated scenario of variation in mutation rates 282 between African and European populations.

283 The expected selection coefficient against a mutator allele. Selection against mutator 284 alleles derives from the excess deleterious germline mutations that individuals with these 
alleles carry. Kimura (1967) approximated the selection coefficient against mutator alleles based on the rate at which extra deleterious mutations are introduced and the rate at which they are removed by selection, recombination, and Mendelian segregation.

Specifically, an individual carrying a single mutator allele produces gametes with an expected $L \phi$ extra de-novo deleterious mutations. Assuming free recombination and Mendelian segregation, we expect half of the extra mutations transmitted with the mutator

291 allele in the previous generation to be transmitted with it to the next generation. Under

292 these assumptions, the number of extra mutations that are linked to a mutator alleles that

293 arose $t$ generations ago, $X(t)$, is Poisson distributed with mean

$$
E(X(t)) \approx L \phi \sum_{i=0}^{t-1}(1 / 2)^{i} .
$$

294 Each mutation stays linked to the mutator allele for two generations on average, so this 295 mean number quickly approaches twice the expected difference in de novo mutations, $296 E(X(\infty))=2 L \phi$. Additionally, each mutation carries a multiplicative fitness loss of $(1-$ $297 h s$ ). When mutator alleles are sufficiently old (e.g., $t>10$ ), then the expected relative 298 fitness of an individual carrying a mutator allele is

$$
E\left((1-h s)^{X}\right) \approx e^{-2 L \phi h s} .
$$

299 suggesting that the expected selection coefficient against mutator alleles is approximately $3001-e^{-2 L \phi h s}$.

301 This approximation becomes inaccurate when selection is very weak or very strong. When 302 selection is weak, mutator alleles can reach high frequencies. High frequency alleles reduce 303 the population's average fitness and thus experience weaker selection, yet they may be 304 found as homozygotes, which experience stronger selection. The combination of these 305 effects reduces the strength of selection by a factor $1-q$ (SI Section 2). When selection is very strong, mutator alleles experience weaker selection for a different reason: they do not

307 persist long enough to accumulate $2 L \phi$ extra mutations. For this case, we calculate the 308 expected strength of selection against mutator alleles, $s^{*}$, accounting for their short 309 lifespans (SI Section 1). We combine both corrections and approximate the selection 310 coefficient of mutator alleles by $s^{\star}(1-q)$, which is accurate throughout the range of 
311 selection effects. Specifically, when selection is strong, $q \ll 1$ and thus $s^{\star}(1-q) \approx s^{*}$ and

312 when selection is weak, $s^{\star} \approx 2 L \phi h s$ (see Fig. S1) and thus $s^{\star}(1-q) \approx 2 L \phi h s(1-q)$.

313 The stationary distribution of mutator alleles. Given the selection coefficient against

314 mutator alleles, we calculate the stationary frequency distribution at the bi-allelic modifier

315 sites. We do so using the diffusion approximation based on the first two moments of change

316 in mutator allele frequency in a single generation (Crow and Kimura 1970; Ewens 2004).

317 We separate the $1^{\text {st }}$ moment into the expected frequency change due to mutation

$$
E\left(\Delta_{\mu} q\right)=\mu(1-q)+\mu q=\mu(1-2 q),
$$

where the two terms correspond to mutations to and from the mutator allele, respectively,

319 and the expected change due to selection

$$
E\left(\Delta_{s} q\right) \approx\left(q-s^{*}(1-q) \cdot q(1-q)-2 \cdot s^{*}(1-q) \cdot q^{2}\right) / \bar{w}(q)-q \approx-q(1-q)^{2} s^{\star},
$$

where the two middle terms correspond to selection against heterozygotes and homozygotes for the mutator allele, respectively, and $\bar{w}(q)=1-2 q \cdot s^{*}(1-q)$ is the

322 relative mean fitness. Taken together, we approximate the expected change in mutator

323 allele frequency by

$$
E(\Delta q) \approx E\left(\Delta_{S} q\right)+E\left(\Delta_{\mu} q\right) \approx-q(1-q)^{2} s^{\star}+\mu(1-2 q) .
$$

324 In turn, the variance of change in mutator allele frequency (the $2^{\text {nd }}$ moment) is well

325 approximated by the standard drift term:

$$
V(\Delta q) \approx \frac{q(1-q)}{2 N}
$$

326 under the standard assumption that $s^{\star} \ll 1$. Based on these moments, the stationary

327 distribution takes the form:

$$
\Phi(q)=C e^{-4 N s^{\star}(1-\mathrm{q} / 2) q}(q(1-q))^{4 N \mu-1}
$$

328 where $C$ is a normalizing constant such that $\int_{0}^{1} \Phi(q) d q=1$ (Fisher 1923; Wright 1945;

329 Kimura 1955; Ewens 2004). Since $4 N \mu \ll 1$, the integral of the distribution is singular at

330 the boundaries; therefore, we use a standard approach to discretize $\Phi(q)$ near 0 and 1 in

331 order to calculate summaries of interest (Ewens 2004, SI Section 3). This distribution

332 depends only on the two compound parameters: the scaled mutation rate $4 N \mu$ and the

333 scaled selection parameter $4 N s^{\star}$. In the analyses below, we vary the effect size $\phi$, which in 
334 turn changes $4 N s^{*}$ and the strength of selection against mutator alleles. We measure the 335 strength of selection in terms of the scaled selection parameter $4 N h s L \phi$.

336 Expectation and variance of the mutation rate. We use the stationary distribution to 337 approximate the effect of modifier sites on the expectation and variance of the mutation 338 rate. We first consider the effects of a single modifier site. The expected increase in the 339 mutation rate is given by

$$
E(\Delta u)=2 \phi E(q)=2 \phi \int_{0}^{1} q \Phi(q) d q
$$

340 We use the law of total variance to divide the variance in mutation rates into expected 341 variance in mutation rates within a population, $E(V(u \mid q))$, and the variance of the mean

342 mutation rate of a population, $V(E(u \mid q))$, which reflects variance between populations, 343 such that

$$
V(u)=E(V(u \mid q))+V(E(u \mid q)) .
$$

344 The variance within a population is

$$
E(V(u \mid q))=\phi^{2} \int_{0}^{1} 2 q(1-q) \Phi(q) d q=\phi^{2} \cdot \bar{\pi}(\phi),
$$

345 where $\bar{\pi}(\phi)$ is the expected heterozygosity at a modifier site with effect size $\phi$. The

346 variance between populations is

$$
V(E(u \mid q))=V(\bar{u})=4 \phi^{2} V(q \mid \phi),
$$

347 where $\bar{u}$ is the mean rate in the population with a given mutator allele frequency, which we 348 refer to as the mean mutation rate. Most of our analyses focus on variance between

349 populations, $V(\bar{u})$, but we return to the expected variance within a population in the

350 Discussion. Assuming linkage equilibrium (LE) among modifier sites, the expressions for

351 the expectation and variance of mutation rates given $M$ modifier sites become:

$$
\begin{gathered}
E(u)=u_{0}+E(\Delta u)=u_{0}+M \cdot 2 \phi E(q) . \\
V(\bar{u})=M \cdot 4 \phi^{2} V(q \mid \phi) \text { and } \\
E\left(V\left(u \mid q_{1}, \ldots, q_{M}\right)\right)=M \cdot \phi^{2} \bar{\pi}(\phi) .
\end{gathered}
$$

352 Our approximations perform well throughout the range of selection regimes and a range of 353 numbers of modifier sites when compared to simulation results (Fig. 1). 
A. Increase in expected mutation rate from modifiers

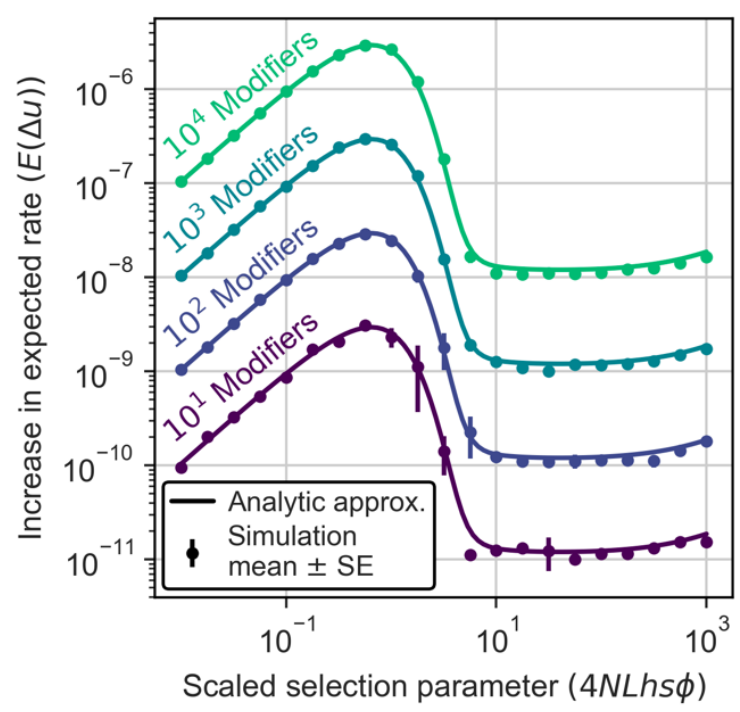

B. Variance in mean mutation rate from modifiers

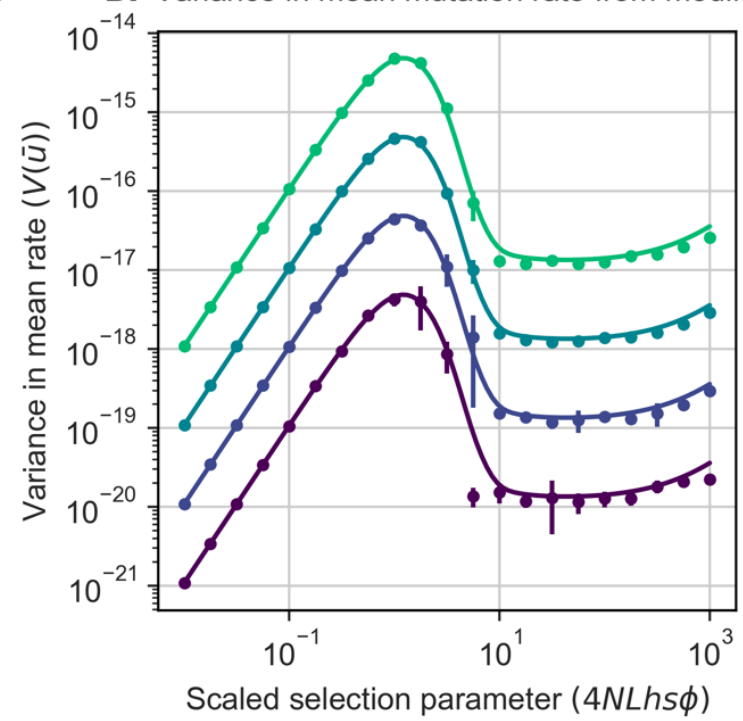

Figure 1. Effect of modifier sites on (A) expected mutation rates and (B) variance of mean mutation rates. We estimate both quantities and their standard error (SE) for each combination of $M$ and the scaled selection parameter $4 N L h s \phi$ (varying $\phi$ ) from simulations (see Simulations). In most cases, the SEs are too small to see and therefore not shown. Analytical results are calculated from Eqs. 13 and 14 . We assume $N=10^{3}$ with other parameters chosen to match population scaled values in humans (see Parameters and Table 1).

The different regimes of modifier dynamics reflect a trade-off between effect size and frequency. A similar trade-off underlies the dependence of genetic load on the selection coefficient (Haldane 1937; Kimura et al. 1963; Kondrashov 1988; Simons et al. 2014). When mutator alleles are effectively neutral $\left(2 N s^{\star} \approx 4 N L h s \phi \ll 1\right)$, selection is too weak to affect allele frequencies. Expected mutation rates therefore increase linearly with $\phi$ and variance in mean rates increase linearly with $\phi^{2}$ (Eqs. 13 and 14); both increases arise primarily from substitutions at modifier sites, half of which are fixed for the mutator allele, rather than from segregating mutator alleles (as $4 N \mu \ll 1$ ). In the nearly neutral regime $\left(2 N s^{\star} \approx 4 N L h s \phi \sim 1\right)$, selection against mutator alleles becomes effective, and fewer sites are fixed for mutator alleles. Effect sizes, $\phi$, in this regime cause the greatest increase in expected rates and variance in mean rates. When selection is strong $\left(2 N s^{\star} \approx 4 N L h s \phi \gg\right.$ 1), modifier sites follow the classic mutation-selection balance model and mutator alleles are restricted to low frequencies. Increases in effect size are countered by a proportional 
375 decrease in frequency yielding approximately constant expected rates and variance in

376 population mean rates (Eqs. 13 and 14). Lastly, when selection becomes very strong (e.g.,

$377 s^{\star}>0.1$ ), $s^{\star}$ is substantially smaller than the selection parameter $2 h s L \phi$ (Fig. S1), and

378 increases in effect size are not fully countered by decreases in frequency, resulting in an

379 increase of the expected rates and variance of population mean rates.

380 Considering the trade-off between effect size and frequency also clarifies how factors we

381 did not include in the model would affect the results. For instance, we assume free

382 recombination among sites. Linkage disequilibrium (LD) between modifier and selected

383 sites would increase the excess number of deleterious mutations linked to mutator alleles

384 relative to the number in our model, which would cause stronger selection against mutator

385 alleles (Kimura et al. 1963; Lynch 2008). As a result, mutator alleles would reach lower

386 frequencies and cause smaller increases in the expectation and variance of mutation rates.

387 The strength of selection would still be proportional to effect size (Kimura 1967), so

388 modifier sites would exhibit similar dynamic regimes to those described. Similarly, we only

389 consider effects of modifier sites on germline mutation rates. If some also increase somatic

390 mutation rates, they likely experience additional selection (e.g., because they increase the

391 incidence of cancer) that increases with their effect size (Lynch 2008). Such modifier sites

392 would have reduced effects on germline mutation rates but plausibly exhibit similar

393 dynamic regimes (Fig. S2). In turn, modifier sites that affect only a subset of possible

394 mutation types affect fewer sites (i.e., smaller $L$ ) compared to less specific modifier sites.

395 When we keep the scaled selection coefficient, $4 N L h s \phi$, constant, more specific modifier

396 sites have a larger effect size, $\phi$, resulting in a greater expected mutation rate and larger

397 variance at affected sites. However, because the increase in effect size is balanced by a

398 decrease in the number of affected sites, modifier sites matched for $4 N L h s \phi$ result in the

399 same increase in the expectation and variance of the total mutation rate.

400 Bounds on the number of modifier sites. Our results suggest that the expectation and 401 variance of mutation rates are proportional to the number of modifier sites (Eqs. 13-15).

402 Investigating the potential variance in mutation rates therefore requires placing bounds on 403 the potential number of modifier sites. One approach is to consider the number and size of 
404 genes involved in DNA replication and repair. In humans, there are 742 unique genes under

405 the Gene Ontology terms DNA replication (G0:0006260) and DNA repair (G0: 0006281)

406 with an average transcript length of $3 \mathrm{~Kb}$, amounting to $\sim 1.8$ million potential modifier sites

407 (Ashburner et al. 2000; The Gene Ontology Consortium 2019). While mutations at many of

408 these sites are likely to be lethal (e.g., those in genes with pLI $\approx 1$ (Lek et al. 2016) or with

$409 h \mathrm{~s} \approx 1$ (Cassa et al. 2017)) or to have no effect on mutation rates (e.g., synonymous), some

410 regulatory sites will affect mutation rates as well. These considerations suggest that there

411 could be as many as hundreds of thousands of modifier sites.

412 As an alternative approach, we bound the potential number of modifier sites by requiring 413 the expected mutation rate $E(u)$ to match the total mutation rate estimate of $1.25 \times 10^{-8}$

414 per bp per generation in humans. When we consider modifier sites that affect only a given

415 mutation type (e.g., TCC $\rightarrow$ TTC), we take the expected rate to be a third of this estimate. If

416 we assume the estimated rate $\hat{u}$ is close to the expectation $E(u)$ and arises entirely from

417 mutation-selection-drift balance at modifier sites (i.e., the baseline rate $u_{0}=0$ ),

418 rearranging Eq. 13 suggests that the maximal number of modifier sites with effect size $\phi$ is

$$
M^{*}(\phi) \approx \frac{\hat{u}}{2 \phi E(q)}
$$

419 Substituting $M^{*}(\phi)$ into Eq. 14, we find that the corresponding variance in mean mutation 420 rates is

$$
V(\bar{u} \mid E(u)=\hat{u}) \approx M^{*}(\phi) \cdot 4 \phi^{2} V(q) \approx 2 \phi \cdot \hat{u} \cdot V(q) / E(q)
$$

421 For brevity, we denote this variance by $V(\bar{u} \mid \hat{u})$. These bounds on the numbers of modifier 422 sites and corresponding variances are shown in Fig. 2. 
A. Bounds on number of modifier sites

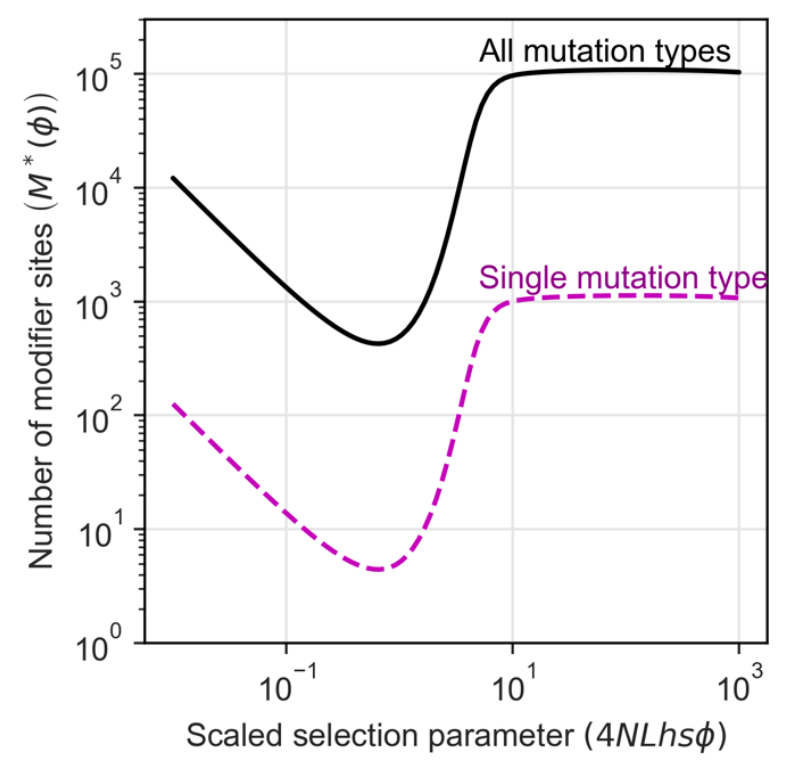

B. Bounds on variance in mean mutation rates

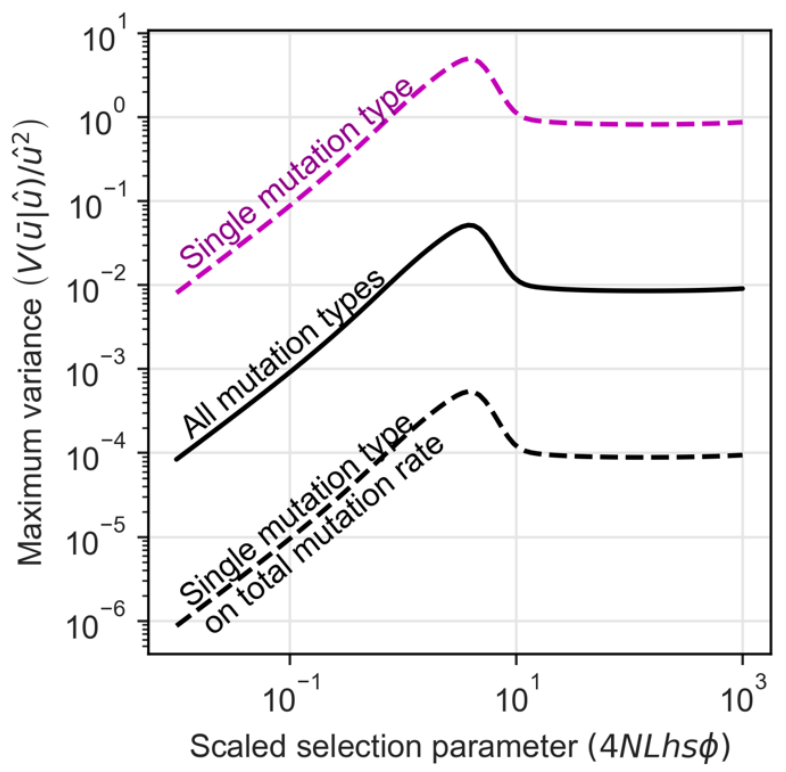

Figure 2. Upper bound on the number of modifier sites (A) and on variance in mean mutation rates (B), assuming that the expected mutation rate equals the estimated rate in humans. We show both quantities for modifier sites that affect one of 96 mutation types (dashed lines) and for those that affect all mutation types (solid lines). For modifier sites that only affect a single mutation type, we show both their effect on variance at sites they affect and on the total mutation rate. Each quantity was calculated using Eqs. 16 and 17. We assumed scaled parameter values roughly based on humans with $N=2 \cdot 10^{4}$ (see Parameters and Table 1).

The variance in mean mutation rates conditional on a fixed expectation exhibits similar selection regimes as it does without this conditioning (Fig. 2). In the effectively neutral regime, selection negligibly affects the frequency of mutator alleles, so $V(q) / E(q)$ remains approximately constant, and $V(\bar{u} \mid \hat{u})$ increases linearly with the effect size, $\phi$ (Eq. 17). When mutators are strongly selected (i.e., under mutation-selection balance), then $E(q) \propto 1 / \phi$ and $V(q) \propto 1 / \phi^{2}$, as $s^{*} \propto \phi$. Consequently, $V(\bar{u} \mid \hat{u})$ is approximately constant. The conditional variance $V(\bar{u} \mid \hat{u})$ is maximized in the nearly neutral regime. Using our estimates of human parameters (Table 1) and the upper bounds on the number of modifier sites $\left(M^{*}(\phi)\right)$, the maximum variance in the total mutation rate (relative to the expected rate squared) is $\sim 5 \%$. However, if modifier sites only affect the rate of one of 96 mutation types, the maximum variance in that focal rate (again relative to the expected rate squared) is $\sim 500 \%$. As expected, varying other parameters that make up the scaled 
444 selection parameter yields similar results (Fig. S2). We also find that this conditional

445 variance behaves similarly when we allow mutator alleles to increase the mutation rate at

446 both selected and modifier sites (Fig. S5).

447 Interpreting the variance in mean mutation rate across populations. Our analytic

448 results overestimate the variance expected among human populations. The variance that

449 we calculate (Eqs. 14 and 17) reflects variation in mean mutation rates at a given point in

450 time among independently-evolving populations at steady state. At steady state, most of

451 the variance due to weakly selected modifier sites arises from differences in the number of

452 fixed rather than segregating mutator alleles (because $4 N \mu \ll 1$ ). However, the timescale of

453 turnover of fixations at weakly-selected modifier sites is on the order of the expected time

454 between neutral substitutions, $1 / \mu$ generations, which is much greater than the split time

455 among human populations. Therefore, we expect few fixed differences at weakly selected

456 modifier sites among human populations. In turn, strongly selected modifier sites never fix

457 for the mutator allele and those that segregate turn over on a timescale proportional to the

458 expected sojourn time, $1 / s^{*}$ generations (Fig. S3). Therefore, we expect strongly selected

459 mutator alleles segregating in different human populations to be largely independent.

460 However, observed mutation rate differences among extant populations typically reflect

461 averages over many generations ancestral to the sample. Given the rapid turnover at

462 strongly selected modifier sites, some of the difference in mean mutation rates may

463 average out. For these reasons, we cannot rely on our analytic results to assess whether

464 evolution at modifier sites can explain differences in mutation rates observed among

465 human populations.

466 Variation among human populations. We therefore rely on simulations to address this

467 question. In the standard model that we implement, each modifier site affects the mutation

468 rate at one of 96 mutation types and thus only affects $1 / 32$ of selected sites. We constrain

469 the number of modifier sites and baseline mutation rate, $u_{0}$, such that the expected

470 mutation rate (at steady state) at each mutation type is $\hat{u}=1.25 \cdot 10^{-8} / 3$ per bp per

471 generation (Eq. 16). We simulate $15 \cdot 10^{3}$ mutator allele trajectories under the S\&D

472 demographic history for African and European populations (Fig. S4; see Simulations for 
473 details). For a given effect size $\phi$ and number of modifier sites $M$, we then resample these

474 mutator trajectories to generate $10^{5}$ sets of 96 mean mutation rate trajectories (one for

475 each mutation type), which include a shared part that occurs in the ancestral population, a

476 part that occurs only in the European population, and a part that occurs only in the African

477 population.

478 We use these sets of mutation rate trajectories to ask whether evolution at modifier sites

479 could cause large, peak-like differences in mutation rates, such as the one observed for

480 TCC $\rightarrow$ TTC mutations in European but not African populations. We reason that a peak

481 would be reported regardless of the mutation type, population, and timing in which it

482 occurred, and therefore examine sets of 96 trajectories jointly. We quantify the "peak

483 pattern" using the two kinds of measures that were applied to human data in previous

484 work.

485 Our first measure is based on the Harris and Pritchard (2017) enrichment statistic, which

486 quantifies population differences in the proportion of segregating sites corresponding to a

487 given mutation type. We mimic both their dataset and analysis as described in Tests for

488 variation in mutation rates between populations. In brief, for each set of mutation rate

489 trajectories, we generate realizations of mutation rate enrichments by dropping mutations

490 on a simulated ancestral recombination graph (ARG) and recording the largest enrichment

491 per set (see SI Section 4). We repeat this procedure for given values of $M$ and $\phi$ to generate

492 an empirical distribution of enrichment values, which we use to estimate the probability

493 that the largest enrichment per set of trajectories exceeds 1.1 (whereas the greatest

494 enrichment estimated by Harris and Pritchard (2017) was approximately 1.16).

495 Our second measure follows Speidel et al. (2019), who estimated the average mutation rate

496 corresponding to 96 different mutation types in log-sized time-intervals along lineages

497 leading to present-day African and European individuals. We do not mimic the Speidel et al.

498 (2019) inference step, but instead we calculate the average mutation rate in 14 non-

499 overlapping log-size intervals (7 per population) along our simulated mutation rate

500 trajectories. We then classify a set of mutation rate trajectories as (Fig. 3D): peak-like if it 
501 includes at least one peak-like mutation rate trajectory but no trajectories with multiple

502 elevations; multi-elevated if any trajectories have multiple elevations; or not-peaked if no

503 trajectories have substantially increased mutation rates (see Tests of variance between

504 populations for details). Finally, we estimate the probabilities that a set of mutation rate

505 trajectories fits a given category for different values of $M$ and $\phi$.
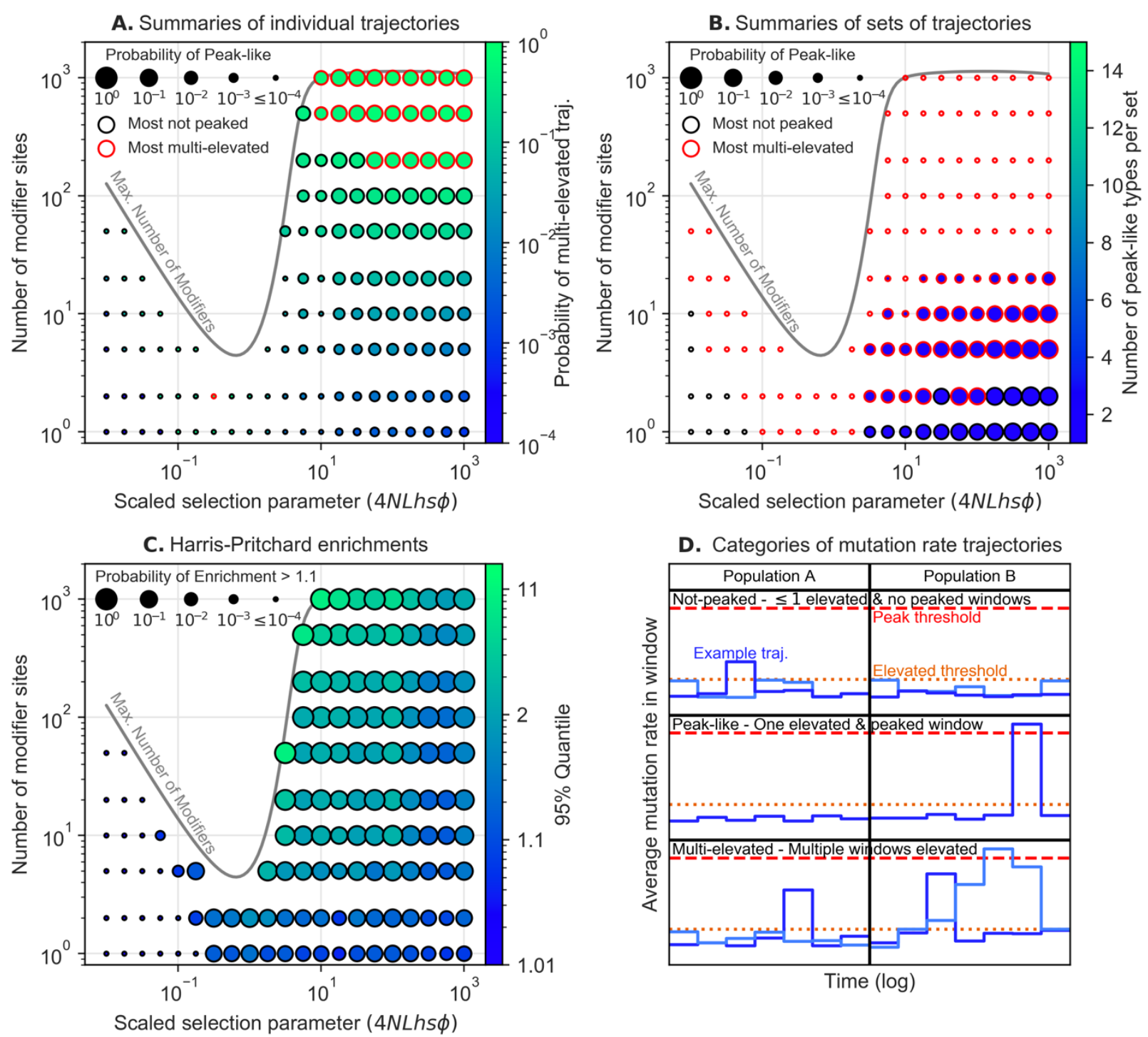

Figure 3. Summary of parameter ranges where modifier sites generate population specific peaks in mutation rates. For a given $M$ and $\phi$, we show the probability that (A) a single mutation rate trajectory or (B) sets of 96 trajectories are peak-like, or the probability that (C) the largest enrichment per set is greater than 1.1 (see Tests of variance between populations). (D) A cartoon illustrating how we categorize trajectories based on the number of windows that are above the elevated or peaked thresholds. For individual trajectories (A), 
513 we show the probability of being multi-elevated (interior color). For sets of trajectories (B),

514 we show the expected number of peak-like trajectories in a peak-like set (interior color). For

515 both (A and B), we show whether most trajectories or sets are not-peaked or multi-elevated

516 (edge color). For enrichments (C), we show the 95\% quantile of enrichment values (interior

517 color). We vary $\phi$ to span the range of selection parameters and vary $M$ between one and the

518 maximum number of modifier sites possible, $M^{*}(\phi)$ (Eq. 16). In Figs. S11-14, we show

519 similar results for models that relax some of our simplifying assumptions.

520 By these approaches, we find that for some parameter ranges, modifier sites frequently

521 generate the differences observed among human populations (Fig. 3). Specifically, a few

$522\left(M \ll M^{*}\right)$ effectively neutral modifier sites ( 4 NLhs $\left.\phi \ll 1\right)$ cause only minor enrichments

523 and almost no peak-like trajectories. However, the cumulative effects of many such

524 modifier sites $\left(M \sim M^{*}\right)$ produces substantial differences in mutation rates between

525 populations, which generate large enrichments, but such sites turn over too slowly to

526 produce substantial differences in rates between populations that are contained to a single

527 time interval. Thus, no number of effectively neutral modifier sites can produce peak-like

528 trajectories. Modifier sites under moderate selection $(1 \leq 4 N \operatorname{Lhs} \phi \leq 10)$ turnover more

529 rapidly and have greater effect sizes (Fig. S3), which allows them to produce larger

530 enrichments and few peak-like trajectories. Modifier sites under stronger selection

531 (4NLhs $\phi>10)$ turnover rapidly enough to allow for both large enrichments and frequent

532 peak-like trajectories. Increasing the number of such modifier sites increases the frequency

533 and magnitude of enrichments as well as the frequency of both peak-like and multi-

534 elevated trajectories. Consequently, too many such modifier sites per mutation type (e.g., >

53520 ) cause large enrichments but also almost always cause multi-elevated sets of

536 trajectories. Putting these observations together, we conclude that a moderate number $(\leq$

53710 per mutation type) under moderate to strong selection can generate large and localized

538 increases in mutation rates that result in both large enrichments and peak-like sets of

539 trajectories.

540 Evolution at modifier sites can also give rise to the observed enrichments and peaks under

541 extensions of our basic model in which we consider: 1) mutation types with different

542 expected rates (e.g., mutations at CpG sites whose rate is an order of magnitude greater 
543 than at other sites (Nachman and Crowell 2000; Kondrashov 2003; Kong et al. 2012)) (Fig.

544 S12), 2) mutator alleles that are selected for their effects on somatic as well as germline

545 mutation rates (Fig. S13); and 3) modifier sites that affect multiple mutation types (e.g.,

546 preferentially affecting 6 out of the 96 possible mutation types), which reflects the fact that

547 different mutation types can arise from common underlying processes (Helleday et al.

548 2014; Alexandrov et al. 2020; Seplyarskiy et al. 2020) (Fig. S14). The parameter ranges

549 with both large enrichments and peak-like sets of trajectories vary somewhat among

550 models, and they vary noticeably with our criteria for peak-like trajectories (Fig. 4, see Fig.

551 S11 for definitions of peak-like). When we use a more relaxed definition of peak-like

552 trajectories (compared to the one used in Fig. 3), the parameter ranges expand but are still

553 restricted to those under moderate to strong selection, which allow for sufficiently rapid

554 turnover. 
A. Basic model

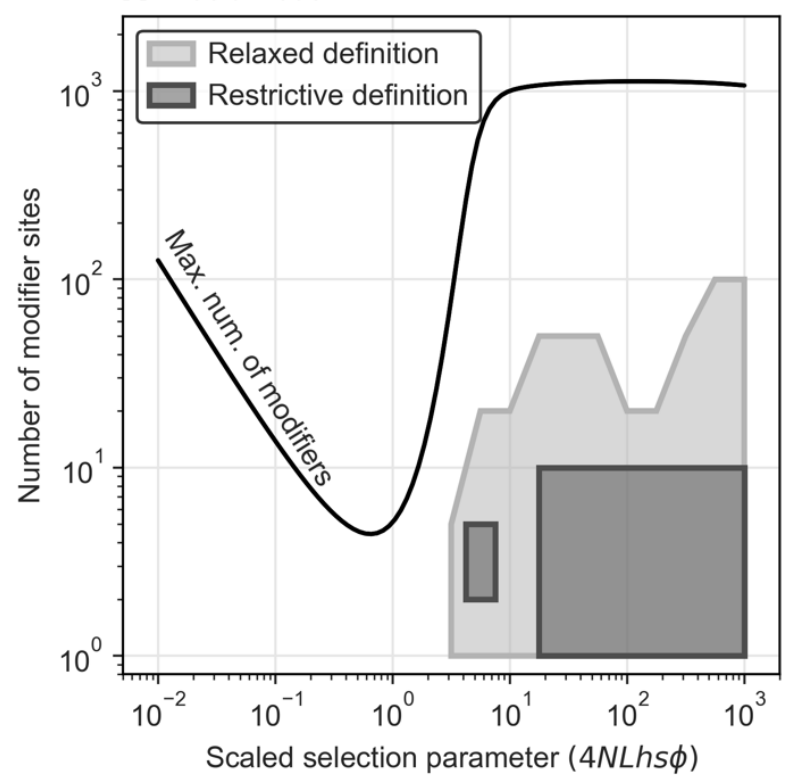

C. Mutators also experience somatic selection

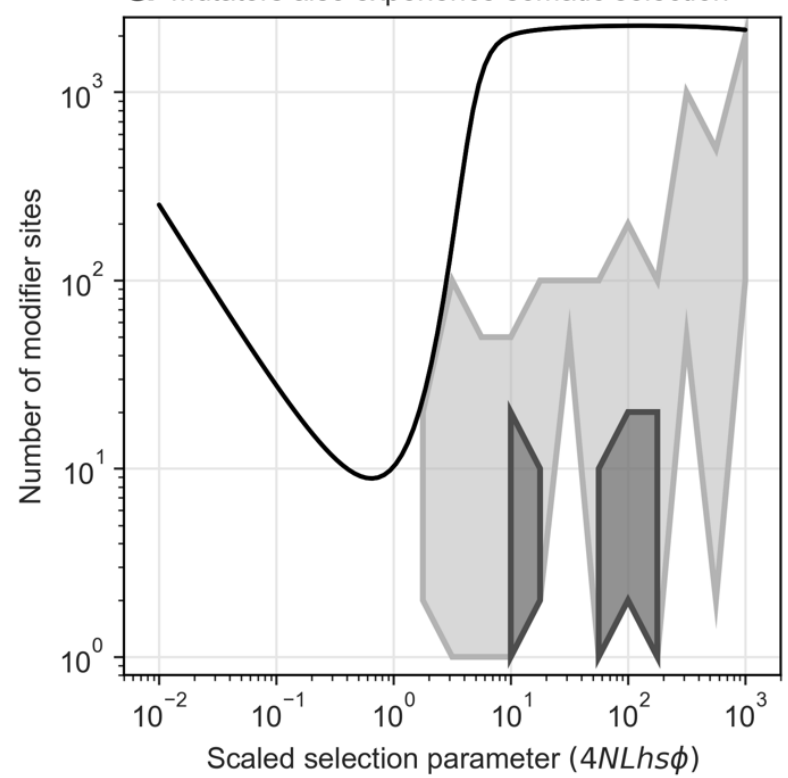

B. Mutation types with different expected rates

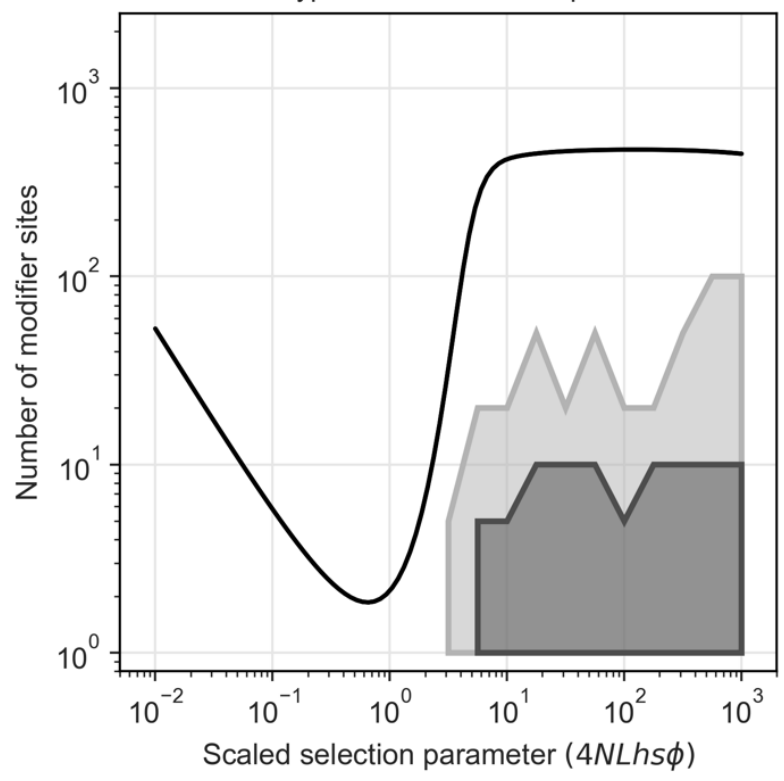

D. Mutators affect multiple mutation types

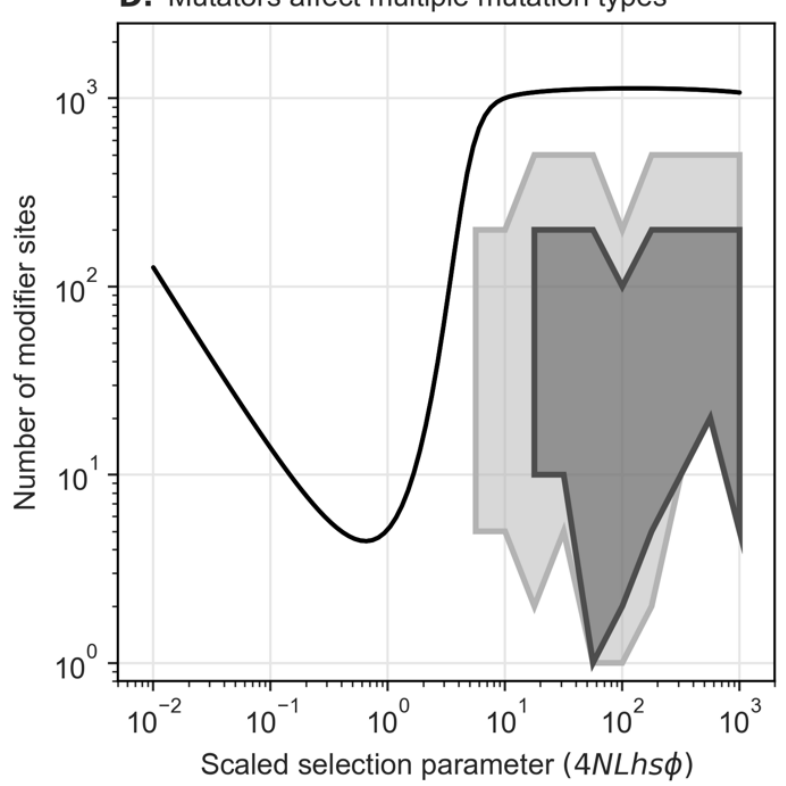

Figure 4. Parameter ranges in which both enrichments and peak-like sets occur with probability $\geq 0.01$ under different models and criteria for peak-like trajectories. $(A)$ Our basic model. (B) A model in which mutation types have different expected rates. (C) A model in which mutator alleles are selected on due to their effects on germline and somatic mutation rates. (D) A model in which mutator alleles affect multiple mutation types. For all models, we show the parameter ranges for our restrictive (dark grey) and relaxed (light grey) definitions of peak-like trajectories (See Tests of variance between populations and Fig. S11 for definitions). See Figs. S12-S14 for additional details about and results for each model (akin to Fig. 3). We calculate the maximal number of modifier sites for a given scaled selection parameter (which varies across models) from Eq. 16. We calculated probabilities 
566

567

as we did for the basic model (see Tests for variation in mutation rates between populations and Fig. S11).

Our analyses suggest that the variation in mutation rates and spectra among human populations could have arisen predominantly from the evolution at modifiers (potentially in addition to changes in life history traits and environmental exposures; as proposed in e.g., Harris 2015; Mathieson and Reich 2017; Macià et al. 2021). This variation arises from the randomness of genetic drift and mutation at modifiers, which causes transient fluctuations in mutation rates and allows otherwise similar populations to evolve different mutation rates. Specifically, evolution at an intermediate number of modifier sites under moderate to strong selection (e.g., $M \leq 10$ per type and $4 N L h s \phi>1$ ) could have caused both the enrichments and peak-like increases in mutation rates observed between African and European populations (Harris 2015; Moorjani et al. 2016; Harris and Pritchard 2017; Mathieson and Reich 2017; Narasimhan et al. 2017; Aikens et al. 2019; Speidel et al. 2019; Goldberg and Harris 2019). Thus, our modeling recasts the question about the role of genetic modifiers in terms of their genetic architecture-i.e., about whether such parameter values are plausible.

We can potentially learn about the genetic architecture by studying mutation rate variation within populations. Unlike differences among populations, variation within a population arises solely from segregating modifier sites rather than substitutions. Specifically, the expected contribution of a single modifier site to variance in mutation rates is $\phi^{2} \cdot \bar{\pi}(\phi)$ (Eq. 11), where $\phi$ is the effect size and $\bar{\pi}(\phi)$ is the expected heterozygosity at the site. To understand how this contribution depends on modifier effect size, we consider simple approximations in the two selection extremes. For effectively neutral modifier sites, $\bar{\pi}(\phi) \approx \theta$, where $\theta=4 N \mu$ is the expected neutral heterozygosity; their expected contribution to variance therefore increases with $\phi^{2}$ and is approximately $\phi^{2} \cdot \theta$. For strongly selected modifier sites, $\bar{\pi}(\phi) \approx 2 E(q)$, where $E(q) \approx \mu / 2 L h s \phi$ is the expected frequency of the mutator allele at mutation-selection balance. Their expected contribution 
to variance therefore increases linearly with their effect size $\left(\phi^{2} \cdot \bar{\pi}(\phi) \approx(\mu / L h s) \cdot \phi\right)$, and the greatest contribution per site arises from large effect and thus rare segregating mutators (Fig. 5A). When we allow for the maximal number of modifier sites given a fixed expected mutation rate, $M^{*}(\phi)$, strongly selected modifier sites still contribute the most to variance within populations (Fig. 5B). This variance may allow us to identify the footprints of such strongly selected modifier sites, and thus we may learn about their number and effect size.

A. Variance from one modifier site

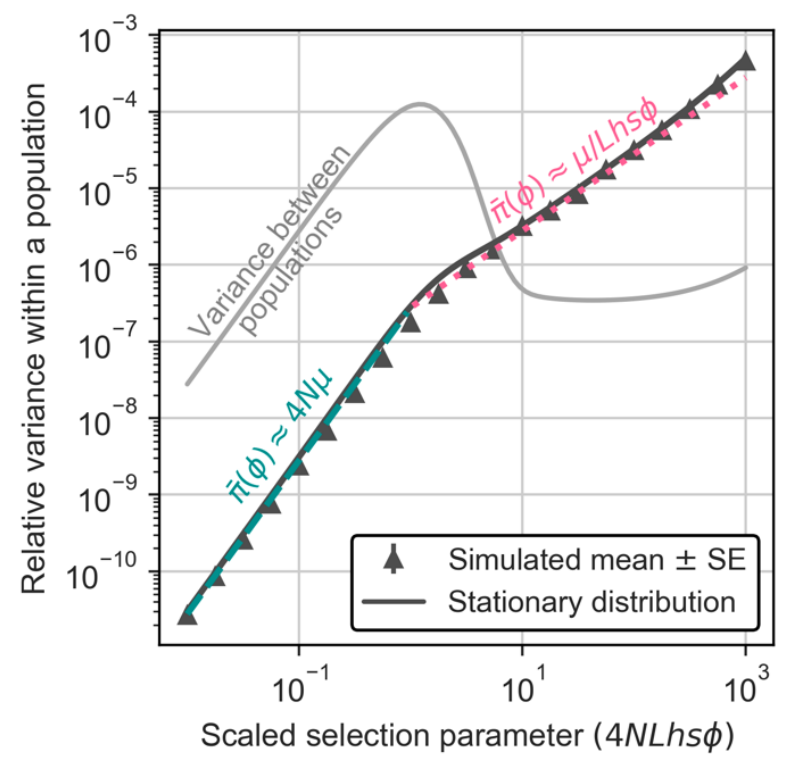

B. Variance from max. number of modifier sites

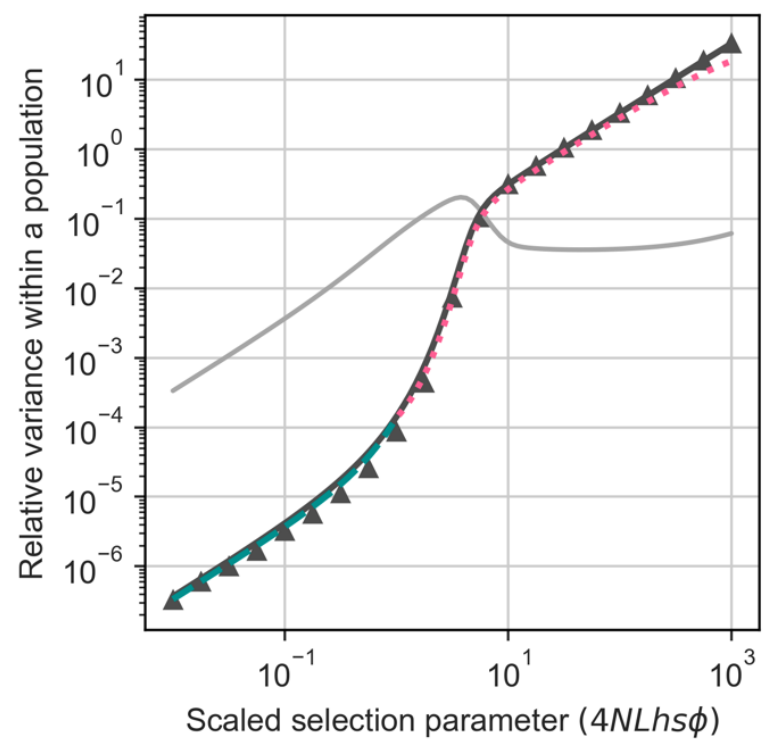

Figure 5. Variance in mutation rates within a population as a function of the effect size of modifier sites. We show the expected variance within a population (relative to a fixed expected rate, $E(V(u \mid q)) / E(u)^{2}$ ) at steady state arising from: (A) one modifier site and (B) the maximum number of modifier sites conditional on a fixed expected rate (Eq. 16). For simulated results with a single site (A), we estimate the average heterozygosity at modifier sites in simulations with $M=10^{3}$ (calculated as described in Simulations) and the contribution to variance within a population relying on Eq. 15. When assuming the maximum number of modifier sites (B), this estimated contribution and its SE are scaled by $M^{*}(\phi)$ 609 (relying on Eq. 16 and assuming $\hat{u}=1.25 \cdot 10^{-8} / \mathrm{bp} / \mathrm{gen}$ ). In most cases, the SEs are too small to see. We calculate the analytical predictions from the expected heterozygosity, which is calculated by integrating over the stationary distribution, and relying on Eq. 15; we also

612 show the results of the simple approximations described in the text. For comparison, we 613 show the corresponding variance between populations, relying on Eq. 14. Both simulated 614 and analytical results assume $N=10^{3}$ with other parameters chosen to match population- 
615 scaled values in humans (see Parameters and Table 1). These results are insensitive to

616 plausible variation in model parameters (Fig. S2).

617 Seoighe and Scally (2017) suggested that mutator alleles segregating within a population

618 could be identified based on the excess mutations linked to them. Closely linked sites are

619 transmitted with the mutator allele and thus experience elevated mutation rates for more

620 generations than loosely linked sites. However, a trade-off between the lifespan of a

621 mutator allele and its effect size limits the expected number of mutations linked to mutator

622 alleles such that this approach is likely underpowered (as we detail in SI Section 5).

623 An alternative approach may be to identify the footprints of strongly selected mutator

624 alleles in pedigree studies of human mutation rates. We expect a parent that is

625 heterozygous for a mutator allele with effect size $\phi$ to pass on $\phi \delta L_{G}$ extra, de novo

626 mutations to their children, where $L_{G}$ is the number of sites (both selected and neutral) in

627 the genome and $\delta$ is the proportion of sites within the genome affected by the mutator

628 allele. If the mutator preferentially affects specific mutation types, it would also change the

629 mutation spectrum. The effects of such a mutator could be detected if: 1) the excess

630 number mutations that it causes is sufficiently large to be identified against the background

631 of other sources of variation in the study, and 2) a parent carrying it is sampled in the

632 study. For the purpose of illustration, we assume our basic model with a constant

633 population size and plausible human parameter values (Table 1), that all de novo

634 mutations are correctly identified, and that the number of de novo mutations in a proband

635 is Poisson distributed with mean $L_{G}\left(u_{M}+u_{P}\right)$, where $u_{M}$ and $u_{P}$ are the maternal and

636 paternal mutation rates. Thus, a proband with parents that carry a single mutator allele

637 with effect size $\phi$ is expected to inherit $L_{G}\left(2 u_{0}+\delta \phi\right)$ de novo mutations. For illustration,

638 we test for an increase in the number of de novo mutations in a trio under a simple null

639 hypothesis, where there are no modifier sites (i.e., $M=0$ ) and the number of de novo

640 mutations is Poisson distributed with mean $2 L_{G} u_{0}$ (here $u_{0}=\hat{u}$ ). Under these assumptions,

641 we expect that a proband with parents that carry a single mutator with $4 N h s L \phi \geq 15$

642 would have a significantly greater number of de novo mutations than expected (with $p<$

643 0.05), and that the significance level will increase with effect size (Fig. 6A). A single 
644 segregating mutator allele with such a large effect would be strongly selected against and

645 rare. Specifically, given a sample of 1500 trios, on par with current human pedigree studies

646 Jónsson et al. 2017; Goldmann et al. 2018; Kessler et al. 2020; Rodriguez-Galindo et al.

647 2020), it is unlikely to be sampled (see SI Section 6). However, if the number of modifier

648 sites is sufficiently large, then one or more mutators are likely to be sampled (Figs. 6B and

649 S15, see SI Section 6 for more details). These considerations suggest that in principle, we

650 should be able to identify the effects of mutators for a wide range of numbers $(M)$ and

651 effect sizes $(\phi)$ of modifier sites with current study designs (Fig. 6).

A. Large effect mutators are detectable

Excess de novo mutations per mutator allele $10^{1}$

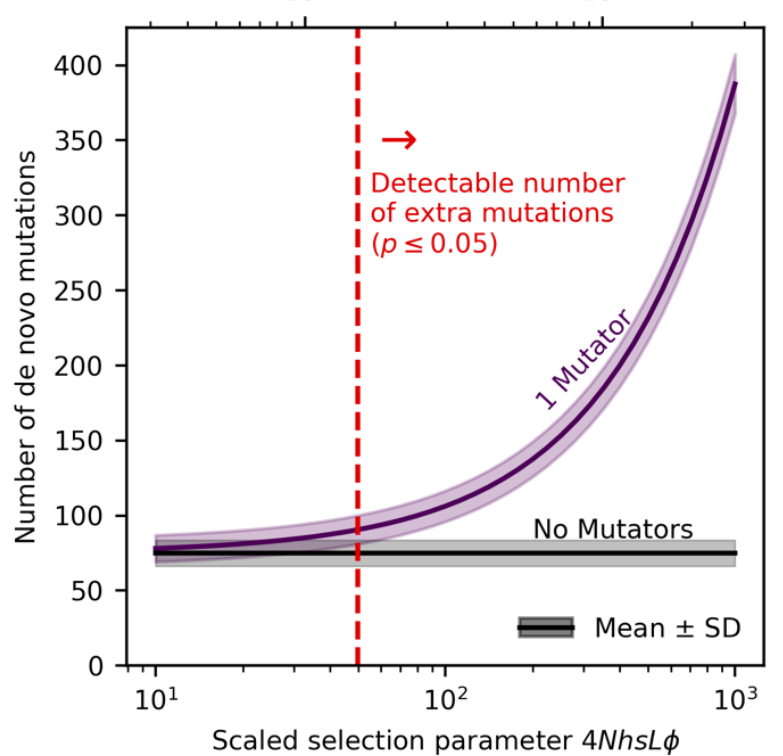

B. Number of mutators sampled in 1500 trios

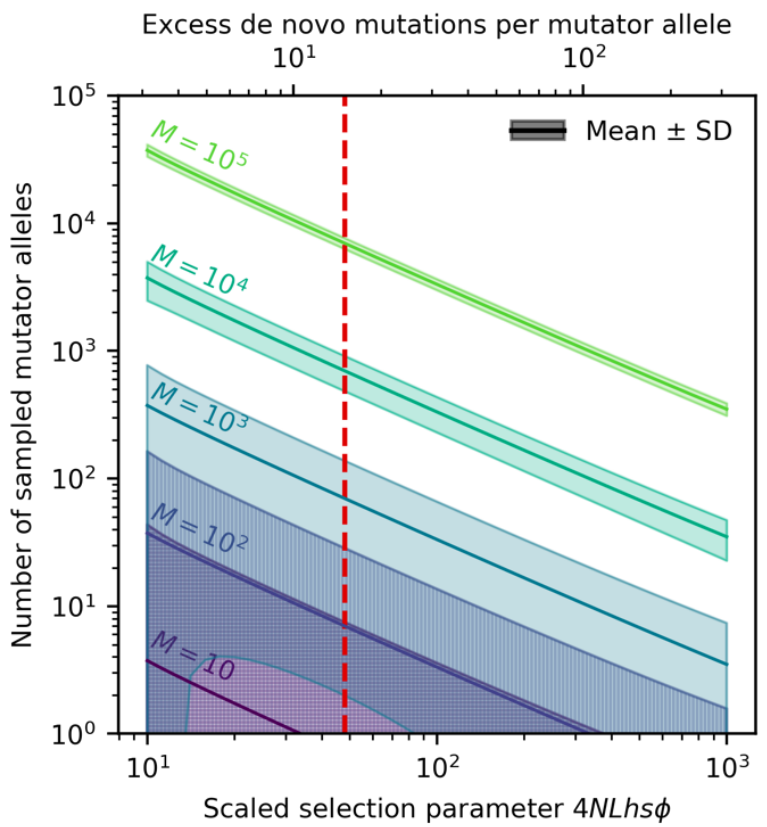

652

653

654

655

656

657

658

659

660

661

Figure 6. Identifying the effects of mutator alleles in a pedigree study of mutation rates. A) The expected number of de novo mutations ( \pm 1 SD) in trios with and without a mutator. B) The expected number of mutators sampled ( \pm 1 SD) in a study with 1,500 trios, based on our steady state approximations (Eq. 8 and SI Section 6). The vertical red lines denote the minimum effect size at which we expect a mutator to be detectable (with $p<$ 0.05). For the purpose of this illustration, we assume a simple null model of mutations in the absence of mutators and ignore potential complications. We further assume that mutators affect all mutations (instead of, e.g., a specific mutation type) and rely on parameter values that are plausible for humans (Table 1) while varying $\phi$. 
662 We expect this qualitative conclusion to hold when we relax our simplifying assumptions.

663 For example, while we rely on our steady state approximations, the expected number of

664 sampled mutator alleles should be insensitive to recent population growth (Simons et al.

665 2014; Simons and Sella 2016). Other sources of mutation rate variation that we have

666 ignored (e.g., parental age effects) could potentially be accounted for by a more realistic

667 null model. Errors in identifying mutations in pedigree studies decrease the power to

668 identify the effects of mutators, but we still expect them to be identifiable if our sample

669 sizes or mutator effect sizes are sufficiently large. Thus, our calculations suggest that the

670 footprints of strongly selected mutators will likely be detectable as pedigree study sample

671 sizes increase allowing us to learn about mutator effect sizes and prevalence.

672 One of our main simplifying assumptions throughout was to ignore the selection on

673 mutator alleles that arises from their effect somatic mutation rates. The form and strength

674 of this selection should depend on 1) the effect of mutators on somatic mutation rates and

675 2) the effect of somatic mutations on fitness, both of which are poorly understood. Somatic

676 and germline mutations arise from an interplay of damage, repair, and replication, which

677 vary among cell lineages (Gao et al. 2016; García-Nieto et al. 2019; Alexandrov et al. 2020).

678 Consequently, the effect of mutator alleles on somatic mutation rates are expected to vary

679 across tissues and cell types (e.g., across neurons, colon crypts, and sperm). The fitness

680 effects of somatic mutations may also be non-trivial. For example, the multi-hit model of

681 carcinogenesis postulates that individual mutations in cancer driver genes may be

682 tolerated whereas multiple mutations cause cancer (Knudson 1971; Knudson 2001;

683 Anandakrishnan et al. 2019), which would suggest a non-linear relationship between

684 somatic mutation rates and fitness (see, e.g., Lynch 2008). We can consider the potential

685 effects of somatic selection on the evolution at modifier loci qualitatively. In the extreme

686 case in which the somatic effects of mutator alleles are lethal, these alleles would have no

687 chance to affect germline mutation rates. In the other extreme, in which selection is

688 predominated by the effects in germline, we would largely recover our results without

689 somatic selection. In between these extremes, the importance of somatic selection depends

690 on its magnitude relative to germline selection, where this relative magnitude may vary

691 with mutator effect size (see, e.g., Lynch 2008). In Fig. S14, we consider a simple model in 
692 which we assume that the strength of selection due to somatic and germline effects is

693 similar. In that case and more generally, the addition of somatic selection reduces the

694 frequency of mutator alleles and thus their impact on the expected mutation rate and the

695 variance in rates within and between populations (Eqs. 13-15, Fig. S2). In turn, the reduced

696 effect of individual modifier sites also allows for a greater number of modifier sites

697 conditional on a given estimated mutation rate (Eq. 16), where the reduced effect and

698 greater number of modifier sites may cancel out to still allow for modifier sites to explain

699 the observed variation among populations and potentially to be identified in pedigree

700 studies (Fig. S14). While these considerations suggest that our qualitative conclusions

701 about variation in germline mutations within and among populations should hold, the

702 potential effects of somatic selection on evolution at modifier loci merit their own study,

703 Conclusion. Further extensions notwithstanding, our work lays the foundations for

704 understanding of how genetic modifiers of germline mutation rates evolve across short

705 evolutionary timescales and affect variation in mutation rates within and among

706 populations. We derived closed forms for these effects for a population of constant size at

707 steady state, which allowed us to characterize how they depend on basic evolutionary

708 parameters. We investigated whether modifier sites can account for the properties of the

709 mutation spectra observed in European and African populations, such as the observed peak

710 in rates of TCC $\rightarrow$ TTC mutations in Europeans. Although we cannot rule out alternative

711 hypotheses for the observed mutation rate variation, our findings suggest that a moderate

712 number of modifier sites subject to moderate to strong selection could account for these

713 patterns and that such modifier sites may soon be identified through sufficiently well-

714 powered pedigree studies of mutation in humans.

715 Acknowledgements. We thank Molly Przeworski for helpful discussions throughout this

716 work. We also benefited greatly from discussion with Ipsita Agarwal, Peter Andolfatto, Laura

717 Hayward, and Felix Wu and from comments on the manuscript from Molly Przeworski. This

718 material is based upon work supported by the National Science Foundation Graduate

719 Research Fellowship under Grant No. DGE1644869. 


\section{References}

721

722

723

724

725

726

727

728

729

730

731

732

733

734

735

736

737

738

739

740

741

742

743

744

745

746

Aarnio M, Sankila R, Pukkala E, Salovaara R, Aaltonen LA, et al. 1999. Cancer risk in mutation carriers of DNA-mismatch-repair genes. Int J Cancer. 81(2):214-218. doi:10.1002/(SICI)1097-0215(19990412)81:2<214::AID-IJC8>3.0.CO;2-L.

Aikens RC, Johnson KE, Voight BF. 2019. Signals of Variation in Human Mutation Rate at Multiple Levels of Sequence Context. Mol Biol Evol. 36(5):955-965. doi:10.1093/molbev/msz023.

Alexandrov LB, Kim J, Haradhvala NJ, Huang MN, Tian Ng AW, et al. 2020. The repertoire of mutational signatures in human cancer. Nature 578(7793):94-101. doi:10.1038/s41586020-1943-3.

Anandakrishnan R, Varghese RT, Kinney NA, Garner HR. 2019. Estimating the number of genetic mutations (hits) required for carcinogenesis based on the distribution of somatic mutations. PLOS Comput Biol. 15(3):e1006881. doi:10.1371/journal.pcbi.1006881.

Ashburner M, Ball CA, Blake JA, Botstein D, Butler H, et al. 2000. Gene Ontology: tool for the unification of biology. Nat Genet. 25(1):25-29. doi:10.1038/75556.

Broughton JM, Weitzel EM. 2018. Population reconstructions for humans and megafauna suggest mixed causes for North American Pleistocene extinctions. Nat Commun. 9(1):5441. doi:10.1038/s41467-018-07897-1.

Carlson J, DeWitt WS, Harris K. 2020. Inferring evolutionary dynamics of mutation rates through the lens of mutation spectrum variation. Curr Opin Genet. 62:50-57. doi:10.1016/j.gde.2020.05.024.

Cassa CA, Weghorn D, Balick DJ, Jordan DM, Nusinow D, et al. 2017. Estimating the selective effects of heterozygous protein-truncating variants from human exome data. Nat Genet. 49(5):806-810. doi:10.1038/ng.3831.

Chintalapati M, Moorjani P. 2020. Evolution of the mutation rate across primates. Curr Opin Genet. 62:58-64. doi:10.1016/j.gde.2020.05.028.

Crow JF, Kimura M. 1970. An introduction to population genetics theory. Burgess Pub, Minneapolis (Minnesota)

Dawson KJ. 1998. Evolutionarily Stable Mutation Rates. J Theor Biol. 194(1):143-157. doi:10.1006/jtbi.1998.0752.

Drake JW. 1993. General Antimutators are Improbable. J Mol Biol. 229(1):8-13.

Drobetsky EA, Sage E. 1993. UV-induced G:C $\rightarrow$ A:T transitions at the APRT locus of Chinese hamster ovary cells cluster at frequently damaged $5^{\prime}$-TCC-3' sequences. Mutat Res Fundam Mol Mech Mutagen. 289(2):131-138. doi:10.1016/0027-5107(93)90062-K. 
Elango N, Lee J, Peng Z, Loh Y-HE, Yi SV. 2009. Evolutionary rate variation in Old World monkeys. Biol Lett. doi:10.1098/rsbl.2008.0712.

756

757

758

759

760

761

762

763

764

765

766

767

768

769

770

771

772

773

774

775

776

777

778

779

780

781

782

783

784

785

786

787

Elango N, Thomas JW, Yi SV. 2006. Variable molecular clocks in hominoids. PNAS. 103(5):1370-1375. doi:10.1073/pnas.0510716103.

Ewens WJ. 2004. Mathematical Population Genetics 1: Theoretical Introduction. 2nd ed. Springer-Verlag, New York (New York)

Felsenstein J. 1974. The Evolutionary Advantage of Recombination. Genetics 78(2):737756.

Feng C, Pettersson M, Lamichhaney S, Rubin C-J, Rafati N, et al. 2017. Moderate nucleotide diversity in the Atlantic herring is associated with a low mutation rate. eLife 6:e23907. doi:10.7554/eLife.23907.

Firestone RB, West A, Kennett JP, Becker L, Bunch TE, et al. 2007. Evidence for an extraterrestrial impact 12,900 years ago that contributed to the megafaunal extinctions and the Younger Dryas cooling. PNAS. 104(41):16016-16021. doi:10.1073/pnas.0706977104.

Fisher RA. 1923. XXI.—On the Dominance Ratio. P Roy Soc Edinb. 42:321-341. doi:10.1017/S0370164600023993.

Gao Z, Wyman MJ, Sella G, Przeworski M. 2016. Interpreting the Dependence of Mutation Rates on Age and Time. PLOS Biol. 14(1):e1002355. doi:10.1371/journal.pbio.1002355.

García-Nieto PE, Morrison AJ, Fraser HB. 2019. The somatic mutation landscape of the human body. Genome Biol. 20(1):298. doi:10.1186/s13059-019-1919-5.

Goldberg ME, Harris K. 2019. Great ape mutation spectra vary across the phylogeny and the genome due to distinct mutational processes that evolve at different rates. bioRxiv. doi:10.1101/805598.

Goldmann JM, Seplyarskiy VB, Wong WSW, Vilboux T, Neerincx PB, et al. 2018. Germline de novo mutation clusters arise during oocyte aging in genomic regions with high doublestrand-break incidence. Nat Genet. 50(4):487-492. doi:10.1038/s41588-018-0071-6.

Haldane JBS. 1937. The Effect of Variation of Fitness. Am Nat. 71(735):337-349. doi:10.1086/280722.

Harland C, Charlier C, Karim L, Cambisano N, Deckers M, et al. 2017. Frequency of mosaicism points towards mutation-prone early cleavage cell divisions in cattle. bioRxiv. doi:10.1101/079863.

Harris K. 2015. Evidence for recent, population-specific evolution of the human mutation rate. PNAS. 112(11):3439-3444. doi:10.1073/pnas.1418652112. 
Harris K, Pritchard JK. 2017. Rapid evolution of the human mutation spectrum. McVean G, editor. eLife. 6:e24284. doi:10.7554/eLife.24284.

790 Helleday T, Eshtad S, Nik-Zainal S. 2014. Mechanisms underlying mutational signatures in human cancers. Nat Rev Genet. 15(9):585-598. doi:10.1038/nrg3729. human germline de novo mutations in 1,548 trios from Iceland. Nature 549(7673):519-

794 522. doi:10.1038/nature24018.

795

796

797

798

Kelleher J, Etheridge AM, McVean G. 2016. Efficient Coalescent Simulation and Genealogical Analysis for Large Sample Sizes. PLOS Comput Biol. 12(5):e1004842. doi:10.1371/journal.pcbi.1004842.

Kellis M, Wold B, Snyder MP, Bernstein BE, Kundaje A, et al. 2014. Defining functional DNA elements in the human genome. PNAS. 111(17):6131-6138. doi:10.1073/pnas.1318948111.

Kessler MD, Loesch DP, Perry JA, Heard-Costa NL, Taliun D, et al. 2020. De novo mutations across 1,465 diverse genomes reveal mutational insights and reductions in the Amish founder population. PNAS. 117(5):2560-2569. doi:10.1073/pnas.1902766117.

Kimura M. 1955. Stochastic Processes and Distribution of Gene Frequencies Under Natural Selection. Cold Spring Harb Symp Quant Biol. 20(0):33-53.

doi:10.1101/SQB.1955.020.01.006.

Kimura M. 1967. On the evolutionary adjustment of spontaneous mutation rates. Genet Res. 9(1):23-34. doi:10.1017/S0016672300010284.

Kimura M, Maruyama T, Crow JF. 1963. The Mutation Load in Small Populations. Genetics 48(10):1303-1312.

Knudson AG. 1971. Mutation and Cancer: Statistical Study of Retinoblastoma. PNAS. 68(4):820-823. doi:10.1073/pnas.68.4.820.

Knudson AG. 2001. Two genetic hits (more or less) to cancer. Nat Rev Cancer. 1(2):157162. doi:10.1038/35101031.

Kondrashov AS. 1988. Deleterious mutations and the evolution of sexual reproduction. Nature 336(6198):435-440. doi:10.1038/336435a0.

Kondrashov AS. 1995. Modifiers of mutation-selection balance: general approach and the evolution of mutation rates. Genet Res. 66(1):53-69. doi:10.1017/S001667230003439X.

Kondrashov AS. 2003. Direct estimates of human per nucleotide mutation rates at 20 loci causing mendelian diseases. Hum Mutat. 21(1):12-27. doi:10.1002/humu.10147. 
821 Kong A, Frigge ML, Masson G, Besenbacher S, Sulem P, et al. 2012. Rate of de novo

822 mutations and the importance of father's age to disease risk. Nature 488(7412):471-475.

823 doi:10.1038/nature11396.

824 Leffler EM, Bullaughey K, Matute DR, Meyer WK, Ségurel L, et al. 2012. Revisiting an Old 825 Riddle: What Determines Genetic Diversity Levels within Species? PLOS Biol.

826 10(9):e1001388. doi:10.1371/journal.pbio.1001388.

827 Lek M, Karczewski KJ, Minikel EV, Samocha KE, Banks E, et al. 2016. Analysis of protein828 coding genetic variation in 60,706 humans. Nature 536(7616):285-291.

829 doi:10.1038/nature19057.

830 Lindsay SJ, Rahbari R, Kaplanis J, Keane T, Hurles ME. 2019. Similarities and differences in 831 patterns of germline mutation between mice and humans. Nat Commun. 10(1):4053.

832 doi:10.1038/s41467-019-12023-w.

833 Lynch M. 2007. The Origins of Genome Architecture. Sinauer Associates, Inc. Sunderland 834 (Massachusetts)

835 Lynch M. 2008. The Cellular, Developmental and Population-Genetic Determinants of 836 Mutation-Rate Evolution. Genetics 180(2):933-943. doi:10.1534/genetics.108.090456.

837 Lynch M. 2010. Evolution of the mutation rate. Trends Genet. 26(8):345-352.

838 doi:10.1016/j.tig.2010.05.003.

839 Lynch M, Conery JS. 2003. The Origins of Genome Complexity. Science 302(5649):1401840 1404. doi:10.1126/science.1089370.

841 Macià MC, Skov L, Peter BM, Schierup MH. 2021. Different historical generation intervals in 842 human populations inferred from Neanderthal fragment lengths and patterns of mutation 843 accumulation. bioRxiv. doi:10.1101/2021.02.25.432907.

844 Marionnet C, Benoit A, Benhamou S, Sarasin A, Stary A. 1995. Characteristics of UV-induced 845 Mutation Spectra in Human XP-D/ERCC2 Gene-mutated Xeroderma Pigmentosum and 846 Trichothiodystrophy Cells. J Mol Biol. 252(5):550-562. doi:10.1006/jmbi.1995.0519.

847 Mathieson I, Reich D. 2017. Differences in the rare variant spectrum among human 848 populations. PLOS Genet. 13(2):e1006581. doi:10.1371/journal.pgen.1006581.

849 Moorjani P, Amorim CEG, Arndt PF, Przeworski M. 2016. Variation in the molecular clock of 850 primates. PNAS. 113(38):10607-10612. doi:10.1073/pnas.1600374113.

851 Nachman MW, Crowell SL. 2000. Estimate of the Mutation Rate per Nucleotide in Humans. 852 Genetics 156(1):297-304. doi:10.1093/genetics/156.1.297. 
Narasimhan VM, Rahbari R, Scally A, Wuster A, Mason D, et al. 2017. Estimating the human mutation rate from autozygous segments reveals population differences in human mutational processes. Nat Commun. 8(1):303. doi:10.1038/s41467-017-00323-y.

Pino M, Abarzúa AM, Astorga G, Martel-Cea A, Cossio-Montecinos N, et al. 2019. Sedimentary record from Patagonia, southern Chile supports cosmic-impact triggering of biomass burning, climate change, and megafaunal extinctions at $12.8 \mathrm{ka}$. Sci Rep. 9(1):4413. doi:10.1038/s41598-018-38089-y.

Ponting CP, Hardison RC. 2011. What fraction of the human genome is functional? Genome Res. 21(11):1769-1776. doi:10.1101/gr.116814.110.

Rands CM, Meader S, Ponting CP, Lunter G. 2014. 8.2\% of the Human Genome Is Constrained: Variation in Rates of Turnover across Functional Element Classes in the Human Lineage. PLOS Genetics. 10(7):e1004525. doi:10.1371/journal.pgen.1004525. mutation rates on exons versus introns in humans. Nat Commun. 11(1):3304. doi:10.1038/s41467-020-17162-z.

Sasani TA, Ashbrook DG, Lu L, Palmer AA, Williams RW, et al. 2021. A wild-derived antimutator drives germline mutation spectrum differences in a genetically diverse murine family. bioRxiv. doi:10.1101/2021.03.12.435196.

Sayres MAW, Venditti C, Pagel M, Makova KD. 2011. Do Variations in Substitution Rates and Male Mutation Bias Correlate with Life-History Traits? A Study of 32 Mammalian Genomes. Evolution 65(10):2800-2815. doi:10.1111/j.1558-5646.2011.01337.x.

Scally A, Durbin R. 2012. Revising the human mutation rate: implications for understanding human evolution. Nat Rev Genet. 13(10):745-753. doi:10.1038/nrg3295.

Schiffels S, Durbin R. 2014. Inferring human population size and separation history from multiple genome sequences. Nat Genet. 46(8):919-925. doi:10.1038/ng.3015.

Ségurel L, Wyman MJ, Przeworski M. 2014. Determinants of Mutation Rate Variation in the Human Germline. Annu Rev Genom Hum Genet. 15(1):47-70. doi:10.1146/annurevgenom-031714-125740.

881 Seoighe C, Scally A. 2017. Inference of Candidate Germline Mutator Loci in Humans from 882 Genome-Wide Haplotype Data. PLOS Genet. 13(1):e1006549.

883 doi:10.1371/journal.pgen.1006549.

884 Seplyarskiy VB, Soldatov RA, McGinty RJ, Goldmann JM, Hernandez R, et al. 2020.

885 Population sequencing data reveal a compendium of mutational processes in human 886 germline. bioRxiv. doi:10.1101/2020.01.10.893024. 
887 Simons YB, Sella G. 2016. The impact of recent population history on the deleterious 888 mutation load in humans and close evolutionary relatives. Curr Opin Genet. 41:150-158.

889 doi:10.1016/j.gde.2016.09.006.

890 Simons YB, Turchin MC, Pritchard JK, Sella G. 2014. The deleterious mutation load is 891 insensitive to recent population history. Nat Genet. 46(3):220-224. doi:10.1038/ng.2896.

892 Speidel L, Forest M, Shi S, Myers SR. 2019. A method for genome-wide genealogy estimation 893 for thousands of samples. Nat Genet. 51(9):1321-1329. doi:10.1038/s41588-019-0484-X.

894 Sung W, Ackerman MS, Miller SF, Doak TG, Lynch M. 2012. Drift-barrier hypothesis and 895 mutation-rate evolution. PNAS. 109(45):18488-18492. doi:10.1073/pnas.1216223109.

896 The Gene Ontology Consortium. 2019. The Gene Ontology Resource: 20 years and still 897 G0ing strong. Nucleic Acids Res. 47(D1):D330-D338. doi:10.1093/nar/gky1055.

898 Uchimura A, Higuchi M, Minakuchi Y, Ohno M, Toyoda A, et al. 2015. Germline mutation 899 rates and the long-term phenotypic effects of mutation accumulation in wild-type 900 laboratory mice and mutator mice. Genome Res. 25(8):1125-1134.

901 doi:10.1101/gr.186148.114.

902 Venn 0, Turner I, Mathieson I, de Groot N, Bontrop R, et al. 2014. Strong male bias drives 903 germline mutation in chimpanzees. Science 344(6189):1272-1275.

904 doi:10.1126/science.344.6189.1272.

905 Ward LD, Kellis M. 2012. Evidence of Abundant Purifying Selection in Humans for Recently 906 Acquired Regulatory Functions. Science 337(6102):1675-1678.

907 doi:10.1126/science.1225057.

908 Wright S. 1945. The Differential Equation of the Distribution of Gene Frequencies. PNAS. 909 31(12):382-389. 